\title{
Nonmodal Clinical Decision Support and Antimicrobial Restriction Effects on Rates of Fluoroquinolone Use in Uncomplicated Infections
}

\author{
Lundy R. Gunn ${ }^{1}$ Robert Tunney ${ }^{2,3}$ Kimberly Kelly ${ }^{2,4}$ \\ ${ }^{1}$ Department of Pharmacy, Fayetteville VA Medical Center, \\ Fayetteville, North Carolina, United States \\ 2 Department of Pharmacy Practice, Campbell University College of \\ Pharmacy \& Health Sciences, Buies Creek, North Carolina, United States \\ ${ }^{3}$ East Carolina Heart Institute, Vidant Medical Center, Greenville, \\ North Carolina, United States \\ ${ }^{4}$ Department of Pharmacy, Harnett Health System, Dunn, North \\ Carolina, United States
}

Appl Clin Inform 2018;9:149-155.

\author{
Address for correspondence Lundy R. Gunn, PharmD, Department of \\ Pharmacy, Fayetteville VA Medical Center, 2300 Ramsey Street, \\ Fayetteville, NC 28301, United States (e-mail: lundy.gunn@va.gov).
}

\section{Abstract}

Keywords

- clinical decision support

- pharmacy information systems

- alert fatigue

- CPOE
Background Medication alert overrides remain persistently high over the past decade, influenced by factors such as "alert fatigue" and lack of provider acceptance. Objective We compared the aggregate rate of fluoroquinolone (FQ) prescribing for the treatment of acute sinusitis, acute bronchitis, and uncomplicated urinary tract infections (UTIs) in adult inpatients prior to (historical control group) and after (prospective intervention group) implementation of a program requiring indication when ordering FQ antibiotics in combination with a nonmodal best-practice alert regarding the latest U.S. Food and Drug Administration (FDA) recommendations. We then compared rates of prescribing among provider type, severity of infection, and patient age.

Methods Qualified orders were defined as new FQ orders for acute sinusitis, acute bronchitis, and uncomplicated UTI for adult inpatients between July 2016 through September 2016 (control) or November 2016 through January 2017 (intervention). The primary endpoint was a provider-initiated FQ order for a target indication. Secondary endpoints included FQ orders by provider type and patient age. Rates of FQ use among the target indications were compared between groups by chi-square test of independence with Yates' correction in the analysis of the primary endpoint and Fisher's exact test for secondary endpoints.

Results FQ prescribing for acute bronchitis, and uncomplicated UTI occurred at a rate of $86 / 350(24.6 \%)$ and $62 / 394(15.7 \%)$ in the control and experimental groups, respectively $(p=0.0035)$. No patients receiving FQ qualified for a diagnosis of acute sinusitis.

Conclusion A program combining FQ restriction in combination with nonmodal messaging may have decreased the rate of prescribing for acute bronchitis and uncomplicated UTI, although the contributions of each individual element could not be rigorously assessed. received

August 26, 2017

accepted after revision

December 31, 2017
DOI https://doi.org/ $10.1055 / \mathrm{s}-0038-1626726$. ISSN 1869-0327. 


\section{Background and Significance}

The Medicare and Medicaid Electronic Health Record (EHR) Incentive Program has established "meaningful use" requirements for clinical decision support (CDS) with the intent of providing evidence-based interventions to reduce adverse drug events. ${ }^{1}$ Despite implementation of these meaningful use requirements for drug-drug and drug-allergy interaction alerts starting in 2011, analyses of medication order alerts have shown persistently high override rates over the past decade. ${ }^{2}$ The term "signal-to-noise ratio" is often used to refer to the ratio of relevant data to irrelevant data in CDS alerting. Desensitization due to poor signal-to-noise ratios of alerts has been thought to lead to "alert fatigue" increased alert override rates. ${ }^{2-7}$

A recently published meta-analysis revealed only two modifiable factors associated with improving patient outcomes related to CDS: providing advice to both practitioners and patients and requiring practitioners to justify their overrides of advice. ${ }^{8}$ Lack of acceptance of CDS by providers and persistently high override rates raise concerns regarding the long-term impact of modal alerts (e.g., interruptive alerts requiring the user to override a warning before continuing to interact with the EHR) on physician behavior. ${ }^{2}$ Overrides due to alert fatigue may result in clinically relevant data being ignored and potential interventions being missed. ${ }^{4}$ Noninterruptive best-practice messaging, in conjunction with programmatic changes, may offer an alternative which has not been previously investigated.

Aside from CDS, many antimicrobial stewardship efforts to influence antibiotic prescribing practices have focused on restriction protocols. ${ }^{9-11}$ Antimicrobial prescribing with an optimized antimicrobial stewardship program has been found to be effective in increasing compliance with institutional policies, reducing duration of antibiotic treatment, and associated with concomitant decreased levels of bacterial resistance to drug classes such as fluoroquinolones (FQs). ${ }^{9,10,12}$ In May 2016, the U.S. Food and Drug Administration (FDA) advised restricting FQ for acute sinusitis, acute bronchitis, and uncomplicated urinary tract infection (UTI) due to disabling and potentially permanent side effects involving the tendons, muscles, joints, nerves, and central nervous system. ${ }^{13}$

At Harnett Health System (HHS), a rural academic health care system, antimicrobial stewardship (AS) strategies at HHS include prospective audits of antimicrobial use, de-escalation of therapy based on culture and sensitivity data, dose optimization, intravenous (IV) to per os (PO) conversion, and antimicrobial restriction. Levofloxacin and ciprofloxacin are the only FQs available through the HHS formulary regardless of indication. Restricted antibiotics require an indication for use upon ordering and may be initiated with pharmacist review pending infectious disease consult. Provided indications are visible to pharmacists for order review prior to verification. FQ restriction was initiated concurrently with implementation of nonmodal best-practice messaging (e.g., noninterruptive alerts allowing the provider to continue to interact with the EHR) embedded into the ordering process to both advise practitioners and require rationale for antibiotic use by documenting indication.
The impact of an AS strategy combining a restriction with best-practice messaging to providers in a rural health setting has not been reported previously. We therefore sought to examine the composite effect of implementing systematic AS initiatives augmented by targeted nonmodal messaging, specifically in reducing inappropriate FQ orders for uncomplicated infections. We hypothesized that such a program would be effective in optimizing FQ prescribing.

\section{Objective}

We compared the aggregate rate of formulary FQ prescribing among adult patients prescribed antibiotics for the treatment of acute sinusitis, acute bronchitis, and uncomplicated UTI in adult inpatients prior to (control group) and after (experimental group) implementation of a restriction program augmented by targeted nonmodal messaging. We furthermore examined the rate of FQ prescribing patterns by provider type, complication of infection, and patient age.

\section{Methods}

\section{Setting}

HHS is a rural health system, including two teaching hospitals with a total of 151 beds located in Harnett County, North Carolina, United States. HHS uses MEDITECH as its electronic medical record at both hospitals across all inpatient and emergency departments as a means of computerized provider order entry (CPOE) and CDS, medication administration documentation, and laboratory result review.

\section{Design}

This single-center, historical control and prospective cohort study was reviewed and exempted by the Cape Fear Valley Health System Institutional Review Board. FQ orders for adult inpatients admitted between July 2016 through September 2016 (control) or November 2016 through January 2017 (intervention) were adjudicated using the MEDITECH software and screened for eligibility. Nonmodal best-practice messaging was implemented into the EHR by informatics staff and communicated to staff via email, leadership, and through the Pharmacy \& Therapeutics committee. The nonmodal messaging provided a prominent FDA advisement, as shown in - Fig. 1, above the free-text restricted antibiotic field as part of the order process.

Data were extracted through MEDITECH reporting using filtered queries for provider orders by date for levofloxacin formulations (PO and IV) with strengths of 250, 500, or $750 \mathrm{mg}$, or ciprofloxacin formulations with strengths of 200 (IV), 400 (IV), 250 (PO), or $500 \mathrm{mg}$ (PO). Orders from patients with complicated infections, those with one-time emergency department orders not continued as an inpatient, or continuation of home therapy for indication of sinusitis, bronchitis, or uncomplicated UTI were excluded from the analysis. The diagnoses of acute sinusitis, acute bronchitis, or uncomplicated UTI were included based on ICD-10 diagnosis codes (J01, J20, J40, J44, J47, and N39, respectively). Uncomplicated UTI was defined as episodes of acute cystitis or 


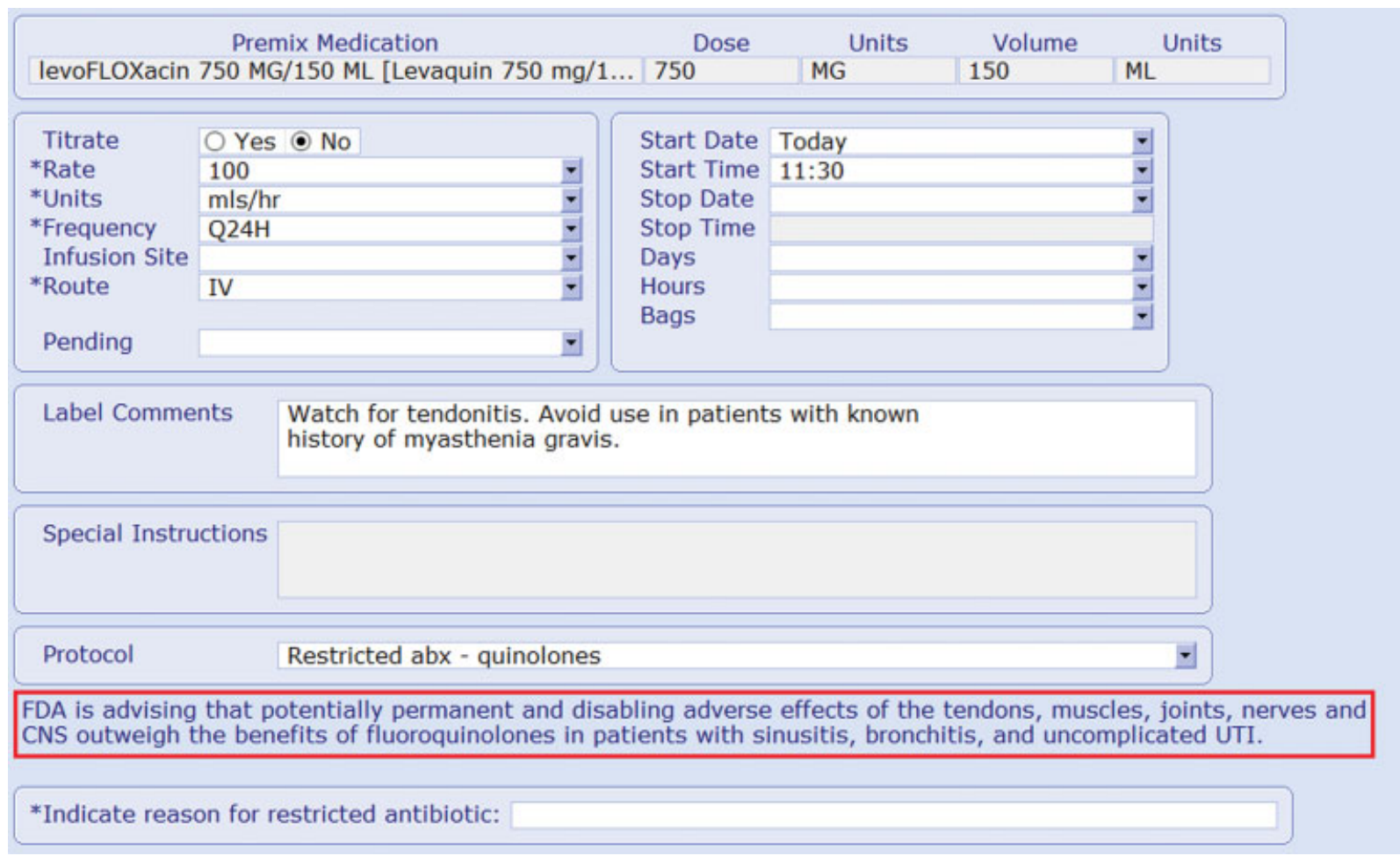

Fig. 1 Screenshot of provider ordering screen, including required indication textbox with nonmodal Food and Drug Administration (FDA) warning message above.

pyelonephritis occurring in healthy premenopausal, nonpregnant women with no history suggestive of an abnormal urinary tract. ${ }^{14}$ Complicated UTI was defined as an infection in the upper or lower urinary tract not meeting criteria for uncomplicated UTI including male patients, catheter-associated infections, critical care unit patients, sepsis, or concomitant bacterial infections other than the genitourinary tract. ${ }^{14}$ Patient visit identifiers were collected for all FQ orders and ICD-10 codes matching selected diagnoses. Patient visit identifiers were then cross-referenced from each list to provide the population of interest. Chart reviews were conducted to clarify diagnoses and verify inclusion criteria.

\section{Data Collection and Analysis}

Data collected included FQ dose, formulation, indication, patient age, and ordering provider. The primary endpoint was defined as the prescribing of a FQ for the target indication (acute sinusitis, acute bronchitis, or uncomplicated UTI). A rate of FQ prescribing was determined among all antibiotic orders for the target indications within the group. Secondary endpoints were defined as the rates of FQ orders within each group by provider type, complication, and patient age.

Aggregate rates of FQ orders of the control and prospective cohorts were compared using chi-squared test of independence with Yates' correction to reduce errors of approximation. Secondary endpoints were analyzed using Fisher's exact test. Statistical significance was predefined as a two-tailed $\alpha \leq 0.05$. Medication orders missing indication data were excluded from analysis. Data were analyzed using JMP version 10.0 .

\section{Results}

A total of 744 patients were identified with a diagnosis of acute bronchitis, or uncomplicated UTI with 350 patients and 394 patients in the control and intervention cohorts, respectively. No patients receiving FQ therapy qualified for a diagnosis of acute sinusitis within the data collection period. Patient demographics and descriptions of FQ prescribing (dose, indication, and route) are summarized in -Table 1. Eighty-six and 62 FQ orders were initiated within the cohorts, respectively, and consisted primarily of high-dose parenteral levofloxacin indicated for acute bronchitis (see - Table 1). The rate of FQ prescribing among the study groups are summarized in -Table 2. FQ prescribing for uncomplicated infections decreased from 24.6 to $15.7 \%(p=0.0035)$. As shown in -Fig. 2, FQ orders were at their lowest for acute bronchitis and uncomplicated UTI during the first month following the incorporation of nonmodal best-practice messaging and antimicrobial restriction. Prescribing of cephalosporins, macrolides, and penicillins increased over the same period (see - Table 2). FQ prescribing by patient age, and provider credentials are summarized in -Table 3. Patients receiving FQs were $\geq 51$ years of age in 78/86 (89.7\%) and 54/62 (87.1\%) in the control and intervention cohorts, respectively. Providers with credentials of medical doctor (MD) initiated approximately $80 \%$ of all FQ orders in each cohort. Insufficient 
Table 1 Baseline patient demographics characteristics

\begin{tabular}{|l|l|l|l|}
\hline Parameter & Control cohort $(\boldsymbol{n}=350)$ & Intervention cohort $(\boldsymbol{n}=394)$ & All patients $(\boldsymbol{n}=\mathbf{7 4 4})$ \\
\hline Age $(\mathrm{y})$, mean $( \pm \mathrm{SD})$ & $67.7( \pm 15.0)$ & $67.2( \pm 14.4)$ & $67.2( \pm 14.4)$ \\
\hline Female, $n(\%)$ & $227(64.9)$ & $240(60.9)$ & $467(62.8)$ \\
\hline FQ, $n(\%)$ & & & \\
\hline Ciprofloxacin & $14(16.3)$ & $10(16.1)$ & $24(16.2)$ \\
\hline $200 \mathrm{mg}$ & - & $1(10.0)$ & $1(4.2)$ \\
\hline $250 \mathrm{mg}$ & $1(7.1)$ & - & $1(4.2)$ \\
\hline $400 \mathrm{mg}$ & $8(57.2)$ & $5(50.0)$ & $13(54.2)$ \\
\hline $500 \mathrm{mg}$ & $5(35.7)$ & $4(40.0)$ & $9(37.5)$ \\
\hline Levofloxacin & $72(83.7)$ & $52(83.9)$ & $124(83.8)$ \\
\hline $250 \mathrm{mg}$ & $3(4.2)$ & $2(3.9)$ & $5(4.0)$ \\
\hline $500 \mathrm{mg}$ & $19(26.4)$ & $6(11.5)$ & $25(20.2)$ \\
\hline $750 \mathrm{mg}$ & $50(69.4)$ & $44(84.6)$ & $94(75.8)$ \\
\hline FQ route, $n$ (\%) & & & \\
\hline PO & $24(27.9)$ & $12(19.4)$ & $36(24.3)$ \\
\hline IV & $62(72.1)$ & $50(80.6)$ & $112(75.7)$ \\
\hline Indication, $n(\%)$ & & & $7(0.9)$ \\
\hline Acute sinusitis & $2(0.6)$ & $366(92.9)$ & $685(92.1)$ \\
\hline Acute bronchitis & $319(91.1)$ & $23(5.8)$ & $52(7.0)$ \\
\hline Uncomplicated UTI & $29(8.3)$ &
\end{tabular}

Abbreviations: FQ, fluoroquinolone; IV, intravenous; PO, per os; SD, standard deviation; UTI, urinary tract infection.

Table 2 Incidence of FQ orders following implementation of restriction and nonmodal messaging

\begin{tabular}{|l|l|l|l|}
\hline & $\begin{array}{l}\text { Control cohort } \\
(\boldsymbol{n}=350)\end{array}$ & $\begin{array}{l}\text { Intervention cohort } \\
(\boldsymbol{n}=394)\end{array}$ & $p$-Value \\
\hline $\begin{array}{l}\text { Acute sinusitis, acute bronchitis, and uncomplicated } \\
\text { UTI patients receiving a FQ }\end{array}$ & $86(24.6 \%)$ & $62(15.7 \%)$ & 0.0035 \\
\hline $\begin{array}{l}\text { Acute sinusitis, acute bronchitis, and uncomplicated } \\
\text { UTI patients receiving other therapy }\end{array}$ & $264(75.4 \%)$ & $332(84.1 \%)$ & 0.9002 \\
\hline Cephalosporin & $150(42.9 \%)$ & $166(42.1 \%)$ & 0.0027 \\
\hline Macrolide & $46(13.1 \%)$ & $86(21.8 \%)$ & 0.3228 \\
\hline Penicillin & $37(10.6 \%)$ & $52(13.2 \%)$ & \\
\hline
\end{tabular}

Abbreviations: FQ, fluoroquinolone; UTI, urinary tract infection.

evidence existed to claim statistical association for either of the secondary endpoints of interest.

\section{Discussion}

Documentation of antibiotic indication is one of the core recommendations outlined by the $\mathrm{CDC}$ for hospital AS programs. ${ }^{15}$ Such programs have demonstrated quality improvement with standalone programmatic changes such as the restriction of antimicrobial classes. Reports of vancomycin restriction requiring order rationale and pharmacist intervention decreased inappropriate use from 39 to $16.8 \%$ $(p=0.005) .{ }^{11}$ As Joint Commission expands the scope of AS standards, there may be room to further influence provider behavior by integrating nonmodal best-practice messaging at critical junctures while still preserving workflow integrity.

Nonmodal messaging has also shown promise as an independent, noninterruptive method of delivering bestpractice alerts. A small study of 24 junior doctors found that providers shown a nonmodal alert were 3.2 times less likely to make a prescribing error. ${ }^{16}$ Given the persistently high rate of alert overrides, identifying modalities which convey evidence-based recommendations to providers without creating untoward interruptions in workflow is a necessary step in further optimizing CDS. Reports from work analysis and interviews have shown that nonmodal medical alerts are appreciated by providers as a "safety net" for situations outside the normal scope of their daily practice. ${ }^{17}$ 


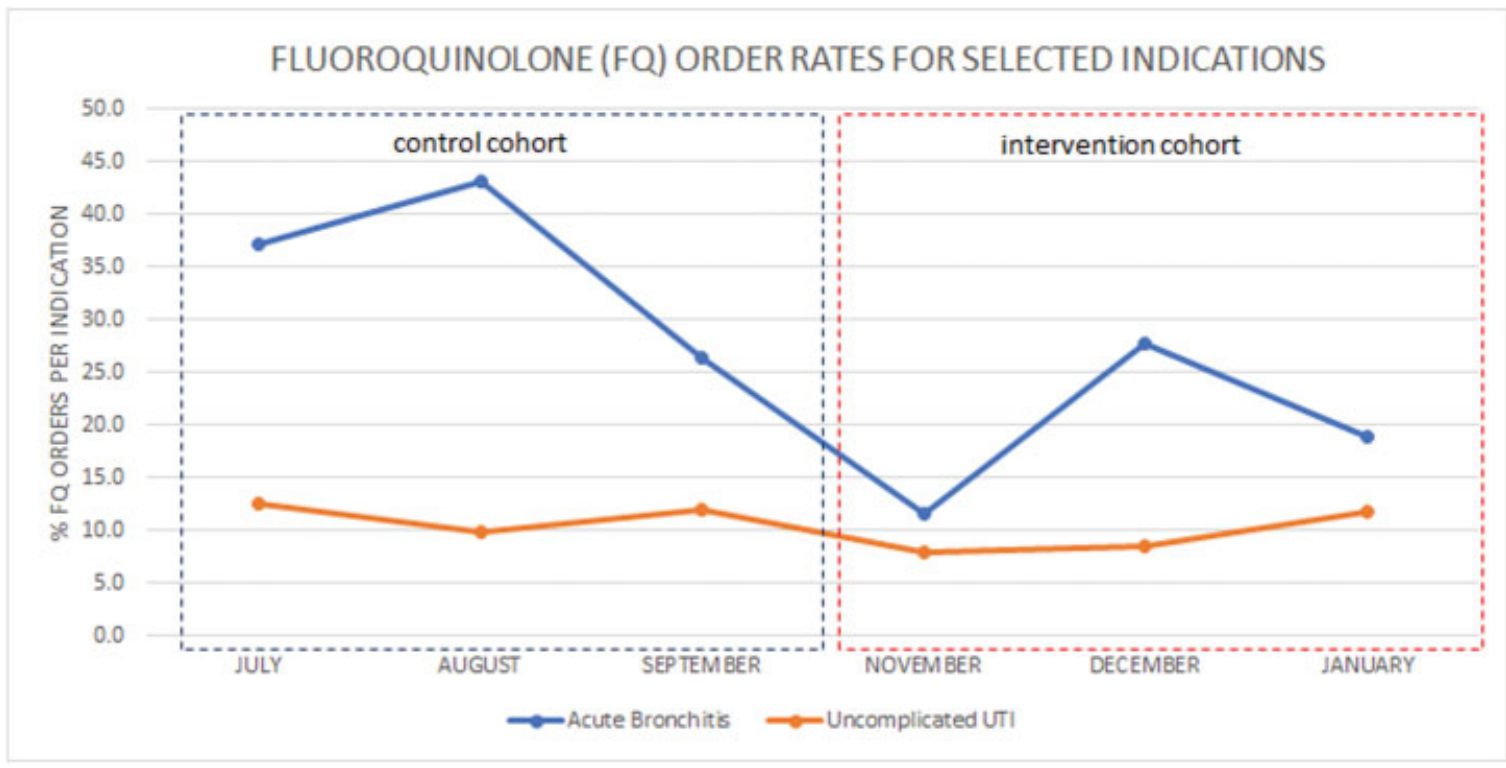

Fig. 2 Monthly fluoroquinolone (FQ) order rates for acute bronchitis and uncomplicated urinary tract infection (UTI).

Table 3 FQ orders by provider type and patient age

\begin{tabular}{|l|l|l|l|}
\hline & Control cohort $(\boldsymbol{n}=\mathbf{8 6})$ & Intervention cohort $(\boldsymbol{n}=\mathbf{6 2})$ & All patients $(\boldsymbol{n}=\mathbf{1 4 8})$ \\
\hline Provider type, $\boldsymbol{n}(\%)$ & & & \\
\hline Doctor of medicine (MD) & $69(80.2)$ & $49(79.0)$ & $118(79.7)$ \\
\hline Doctor of osteopathy (DO) & $8(9.3)$ & $8(12.9)$ & $16(10.8)$ \\
\hline Physician assistant (PA) & $9(10.5)$ & $4(6.5)$ & $13(8.8)$ \\
\hline Nurse practitioner (NP) & - & $1(1.6)$ & $1(0.7)$ \\
\hline Patient age (y), $n(\%)$ & & & $4(2.7)$ \\
\hline $18-30$ & $2(2.3)$ & $2(3.2)$ & $13(8.8)$ \\
\hline $31-50$ & $7(8.0)$ & $6(9.7)$ & $70(47.3)$ \\
\hline $51-70$ & $42(48.3)$ & $28(45.2)$ & $62(41.9)$ \\
\hline$>70$ & $36(41.4)$ & $26(41.9)$ & \\
\hline
\end{tabular}

Abbreviation: FQ, fluoroquinolone.

Considering FDA recommendations, analogous clinical settings should consider performing internal assessments of their AS and best-practice policies related to FQ. Institutions with existing FQ restriction policies may experience added benefit from the introduction of nonmodal alerts within the restricted ordering process. Additional studies are needed to examine the benefit of nonmodal messaging independently of restriction on FQ use in acute bronchitis, acute sinusitis, and uncomplicated UTI.

We evaluated the effect of implementing FQ restriction protocols with embedded nonmodal best-practice messaging. Initial study design sought to examine the effects of FQ restriction and nonmodal messaging separately; however, the informatics team implemented the nonmodal alert in parallel with the restriction policy. The protocol was amended to allow for a composite intervention due to the unforeseen timing of the restriction policy implementation which was not realized until data collection had already commenced. FQ orders for acute bronchitis, acute sinusitis, or uncomplicated UTI decreased from 24.6 to $15.7 \%$ following implementation of nonmodal messaging and antibiotic restriction. The composite effects of nonmodal messaging combined with antibiotic restriction on clinician prescribing behavior have not been examined in the current literature.

There are several potential limitations within this investigation. Chiefly, there existed an inability to directly account for the influence of each element of the primary outcome on end results. There were no patients receiving FQ for acute sinusitis and limited numbers of patients receiving such therapy for uncomplicated UTI. Due to delays and technical failures within the initial study design, we were unable to study each element of the primary outcome independently. There furthermore may have been a time bias as it relates to the seasonal influence of antibiotic prescribing patterns for infectious disease states 
such as pneumonia. Cephalosporin, macrolide, and penicillin data were added as a control to help provide further insight into temporal confounding ( - Table 2 ). FQ order rates were also broken down by month to show any stepwise patterns existing pre- and postintervention and to more clearly elucidate temporal changes, as shown in -Fig. 2. Nadirs for both acute bronchitis and uncomplicated UTI occurred in the first month of the intervention. With the exception of a decreasing trend in FQ orders for acute bronchitis, no clear prescribing patterns were discernible potentially due to low numbers of uncomplicated UTI patients meeting inclusion criteria for the study. The observed rise in use of other antibiotics in parallel to decreased FQ use give credence to potential intervention effects rather than seasonal changes. Cohorts were not randomized or matched based on disease severity or previous infections with resistant organisms. Some patient characteristics such as allergies, prior failure, and antibiotic susceptibility that may have justified FQ prescribing were not accounted for. Finally, prescriber satisfaction with the nature of nonmodal alert modalities as a function of streamlining work flow was not robustly assessed.

\section{Conclusion}

Implementation of hospital-wide antibiotic restriction with integrated nonmodal messaging may have reduced FQ prescribing rates for acute bronchitis and uncomplicated UTI. Additional rigorous testing is required to further define the individual effects of the intervention.

\section{Clinical Relevance Statement}

Antimicrobial restriction protocols requiring documentation of specific indications for use are an opportunity to examine nonintrusive CDS options within workflow such as nonmodal messaging. The combination of nonmodal messaging plus restriction has demonstrated a reduced rate of inappropriate prescribing of fluoroquinolones for uncomplicated infections.

\section{Multiple Choice Question}

When implementing CDS to support antimicrobial restriction efforts, which method may effectively influence provider behavior without significantly halting existing clinical workflow?
a. Nonmodal alerts
b. Modal alerts
c. Hard stop alerts
d. Pop-up hyperlink

Correct Answer: The correct answer is a. Nonmodal alerts may decrease inappropriate antibiotic use when combined with antibiotic restriction protocols. Modal alerts and hard stop alerts significantly interrupt workflow. Guideline recommendations provided by hyperlink, while useful, distract from normal workflow by "popping up" and being reduced to a single computer screen.
Protection of Human and Animal Subjects

Human and/or animal subjects were not included in this project, which was reviewed by Cape Fear Valley Health System Institutional Review Board.

\section{Conflict of Interest}

None.

\section{Acknowledgments}

This research was supported by Harnett Health System and Campbell University College of Pharmacy \& Health Sciences. We thank our colleagues from these institutions for their support and feedback throughout the course of this research. We would also like to show our gratitude to a panel of anonymous reviewers for their constructive perspectives.

\section{References}

1 CMS. Home - Centers for Medicare \& Medicaid Services. Centers for Medicare \& Medicaid Services; 2017. Available at: http://www.cms. gov/. Accessed June 19, 2017

2 Bryant AD, Fletcher GS, Payne TH. Drug interaction alert override rates in the Meaningful Use era: no evidence of progress. Appl Clin Inform 2014;5(03):802-813

3 van der Sijs H, Aarts J, Vulto A, Berg M. Overriding of drug safety alerts in computerized physician order entry. J Am Med Inform Assoc 2006;13(02):138-147

4 Forrest GN, Van Schooneveld TC, Kullar R, Schulz LT, Duong P, Postelnick M. Use of electronic health records and clinical decision support systems for antimicrobial stewardship. Clin Infect Dis 2014;59(Suppl 3):S122-S133

5 Saverno KR, Hines LE, Warholak TL, et al. Ability of pharmacy clinical decision-support software to alert users about clinically important drug-drug interactions. J Am Med Inform Assoc 2011; 18(01):32-37

6 Hsieh TC, Kuperman GJ, Jaggi T, et al. Characteristics and consequences of drug allergy alert overrides in a computerized physician order entry system. J Am Med Inform Assoc 2004;11 (06):482-491

7 Schulz L, Osterby K, Fox B. The use of best practice alerts with the development of an antimicrobial stewardship navigator to promote antibiotic de-escalation in the electronic medical record. Infect Control Hosp Epidemiol 2013;34(12):1259-1265

8 Roshanov PS, Fernandes N, Wilczynski JM, et al. Features of effective computerised clinical decision support systems: metaregression of 162 randomised trials. BMJ 2013;346:f657. Doi: 10.1136/bmj.f657

9 Boel J, Andreasen V, Jarløv JO, et al. Impact of antibiotic restriction on resistance levels of Escherichia coli: a controlled interrupted time series study of a hospital-wide antibiotic stewardship programme. J Antimicrob Chemother 2016;71 (07):2047-2051

10 Shea KM, Hobbs ALV, Jaso TC, et al. Effect of a health care system respiratory fluoroquinolone restriction program to alter utilization and impact rates of clostridium difficile infection. Antimicrob Agents Chemother 2017;61(06):e00125-17

11 Richardson LP, Wiseman SW, Malani PN, Lyons MJ, Kauffman CA. Effectiveness of a vancomycin restriction policy in changing the prescribing patterns of house staff. Microb Drug Resist 2000;6 (04):327-330

12 Davey P, Marwick CA, Scott CL, et al. Interventions to improve antibiotic prescribing practices for hospital inpatients. Cochrane Database Syst Rev 2017;2:CD003543 
13 U.S. Food and Drug Administration Home Page. FDA Drug Safety Communication. U.S. Food and Drug Administration. Available at: http://www.fda.gov/downloads/Drugs/DrugSafety/UCM500591. pdf. Accessed June 19, 2017

14 Gupta K, Hooton TM, Naber KG, et al; Infectious Diseases Society of America; European Society for Microbiology and Infectious Diseases. International clinical practice guidelines for the treatment of acute uncomplicated cystitis and pyelonephritis in women: a 2010 update by the Infectious Diseases Society of America and the European Society for Microbiology and Infectious Diseases. Clin Infect Dis 2011;52(05):e103-e120
15 Centers for Disease Control and Prevention. Core Elements of Hospital Antibiotic Stewardship Programs. Atlanta, GA: U.S. Department of Health and Human Services, CDC; 2014. Available at: http://www. cdc.gov/getsmart/healthcare/implementation/core-elements.html. Accessed March 1, 2017

16 Scott GPT, Shah P, WyattJC, Makubate B, Cross FW. Making electronic prescribing alerts more effective: scenario-based experimental study in junior doctors. J Am Med Inform Assoc 2011;18(06):789-798

17 Wipfli R, Betrancourt M, Guardia A, Lovis C. A qualitative analysis of prescription activity and alert usage in a computerized physician order entry system. Stud Health Technol Inform 2011;169:940-944 